\title{
Structural basis of stereospecific reduction by quinuclidinone reductase
}

\author{
Daijiro Takeshita', Michihiko Kataoka, ${ }^{2,3}$ Takuya Miyakawa', Ken-ichi Miyazono', Shoko Kumashiro², Takahiro Nagai \\ Nobuyuki Urano ${ }^{2,3}$, Atsuko Uzura ${ }^{4}$, Koji Nagata', Sakayu Shimizu ${ }^{2,5}$ and Masaru Tanokura ${ }^{\text {** }}$
}

\begin{abstract}
Chiral molecule (R)-3-quinuclidinol, a valuable compound for the production of various pharmaceuticals, is efficiently synthesized from 3-quinuclidinone by using NADPH-dependent 3-quinuclidinone reductase (RrQR) from Rhodotorula rubra. Here, we report the crystal structure of RrQR and the structure-based mutational analysis. The enzyme forms a tetramer, in which the core of each protomer exhibits the $a / \beta$ Rossmann fold and contains one molecule of NADPH, whereas the characteristic substructures of a small lobe and a variable loop are localized around the substrate-binding site. Modeling and mutation analyses of the catalytic site indicated that the hydrophobicity of two residues, 1167 and F212, determines the substrate-binding orientation as well as the substrate-binding affinity. Our results revealed that the characteristic substrate-binding pocket composed of hydrophobic amino acid residues ensures substrate docking for the stereospecific reaction of RrQR in spite of its loose interaction with the substrate.
\end{abstract}

Keywords: Crystal structure; NADPH-dependent enzyme; Asymmetric reduction; 3-Quinuclidinone reductase; (R)-3-quinuclidinol

\section{Introduction}

The chirality of chemical compounds is an important property for their availability as building blocks for pharmaceuticals and chemicals, including chemical catalysts, liquid crystals, flavors, agrochemicals, and medicines. As is often the case, only one optical isomer primarily or completely exhibits the intended activity. Therefore, it is necessary to develop methods to produce optically pure compounds for use in the industrial production of chiral compounds. Biotransformations using whole cells and enzyme-catalyzed reactions have been attempted for the production of various chiral compounds. The enzymatic asymmetric reduction of prochiral carbonyl compounds is a promising method for the production of chiral alcohols, because enzymes have certain advantages for use in chemical reactions under mild conditions and with chemo-, regio-, and stereo-selectivity (Hummel 1997; Kataoka et al. 2003; Goldberg et al. 2007). Structural information about useful enzymes would enable the

\footnotetext{
* Correspondence: amtanok@mail.ecc.u-tokyo.ac.jp

'Department of Applied Biological Chemistry, Graduate School of Agricultural and Life Sciences, University of Tokyo, 1-1-1 Yayoi, Bunkyo-ku, Tokyo 113-8657, Japan

Full list of author information is available at the end of the article
}

generation of new enzymes exhibiting high activity, enhanced stability, and altered-coenzyme specificity, and would also enable elucidation of the detailed reaction mechanisms and substrate-binding specificities.

One of the chiral alcohols, $(R)$-3-quinuclidinol, is a useful compound applicable to the synthesis of various pharmaceuticals, such as talsaclidine and revatropate (Rzeszotarski et al. 1988; Bietti et al. 1990; Cross and Stobie 1993; Takeuchi et al. 1996; Alabaster 1997; Ward et al. 1998; Leusch et al. 2000; Ishihara et al. 2004). So far, chemical and biochemical methods have been applied to produce $(R)$-3-quinuclidinol (Stembach and Kaiser 1952; Rehavi et al. 1977; Muchmore 1993; Bossard 1997; Brieden 1998; Matsuyama and Hamatani 1998; Sato and Enomoto 1999; Nomoto et al. 2003; Yamamoto et al. 2003; Takenaka 2006), and most of these methods take the approach of resolving a racemic mixture. Recently, for the production of $(R)$-3-quinuclidinol, 3-quinuclidinone reductase ( $\mathrm{RQRR}$ ) was isolated from Rhodotorula rubra (Uzura et al. 2009). The purified enzyme from $R$. rubra is specific to NADPH as a coenzyme and stereospecifically produces the $(R)$-enantiomer of 3-quinuclidinol from 3-quinuclidinone. Furthermore, a coexpression system for the 3-quinuclidinone reductase 
and glucose dehydrogenase using Escherichia coli as a host was developed for the large-scale production of $(R)$-3-quinuclidinol. The system using the enzyme is superior to former kinetic resolution methods in that it has a higher molar yield, requires no substrate modification, and so on (Uzura et al. 2009).

Based on the amino acid similarity, RrQR belongs to the short-chain dehydrogenase/reductase family. Though the proteins in this family have low sequence identities, they share a similar $\alpha / \beta$ Rossmann-fold core. In contrast to the fold similarity, oxidoreductases, which make up the majority of the family, exhibit various substrate specificities (Oppermann et al. 2003), indicating that the mechanisms to accommodate structurally different substrates are diverse. The molecular identification and characterization of the enzymes of this family, which have diverse activities capable of producing useful molecules, would be helpful for the utilization of these enzymes. To elucidate the detailed molecular mechanism underlying the stereospecific reduction of the 3-quinuclidinone, we determined the crystal structure of RrQR in complex with NADPH. Modeling and mutation analyses of RrQR indicated a novel mechanism underlying the stereospecific reaction led by a characteristic interaction, explaining how optically pure $(R)$-3-quinuclidinol is synthesized from 3-quinuclidinone.

\section{Materials and methods}

\section{Crystallization and structure determination of RrQR}

RrQR was expressed, purified, and crystallized as described previously (Takeshita et al. 2009). Briefly, the crystals were grown at $278 \mathrm{~K}$ using the sitting-drop vapor-diffusion method with a reservoir solution containing 100 mM CHES (pH 10.0), 30\% PEG8000, and 3\% sucrose. The X-ray diffraction data set was collected at $95 \mathrm{~K}$ at Beamline BL-5A of the Photon Factory, KEK (Tsukuba, Japan). The data were processed using HKL2000 (Otwinowski and Minor 1997). The crystals belonged to space group $P 4_{1} 2_{1} 2$ with unit-cell parameters of $a=b=91.3 \AA$ and $c=265.4 \AA$. The datacollection statistics of the RrQR crystal are summarized in Table 1 and Additional file 1: Table S1. The reason for the lower completeness in the highest resolution shell is the limitation of the camera size at the beamline. The reflection data to $2.2 \AA$ resolution were used for the structural analysis because the values of $R_{\text {merge }}$, redundancy and completeness in the highest resolution shell are valuable for the refinement. The asymmetric unit contained four molecules of RrQR and the RrQR structure was solved by molecular replacement using Phaser (McCoy et al. 2007) with Thermotoga maritima TM0441 (PDB ID 1vl8) as a search model. The model was refined using REFMAC5 (Murshudov et al. 1997) and CNS (Brünger et al. 1998). The model was manually rebuilt

\section{Table 1 Data collection and refinement statistics of the RrQR crystal}

\begin{tabular}{|c|c|}
\hline \multicolumn{2}{|l|}{ Data collection } \\
\hline X-ray source & PF BL-5A \\
\hline Wavelength $(\AA)$ & 1.0000 \\
\hline Space group & $P 4,2,2$ \\
\hline Unit-cell parameters $(\AA)$ & $a=b=91.3, c=265.4$ \\
\hline Resolution $(\AA)^{a}$ & $20.0-2.20(2.28-2.20)$ \\
\hline Observed reflections & 689047 \\
\hline Unique reflections & 55949 \\
\hline Completeness (\%) & $96.5(81.0)$ \\
\hline$R_{\text {merge }}(\%)^{\mathrm{a}, \mathrm{b}}$ & $9.2(23.9)$ \\
\hline$<|>|<\sigma(\mid)\rangle^{a}$ & $55.6(11.4)$ \\
\hline \multicolumn{2}{|l|}{ Refinement } \\
\hline Resolution range $(\AA ̊)$ & $20.0-2.20$ \\
\hline $\mathrm{R}_{\text {factor }}(\%)^{c}$ & 19.2 \\
\hline$R_{\text {free }}(\%)^{d}$ & 23.0 \\
\hline Protein atoms & 8020 \\
\hline NADPH atoms & 192 \\
\hline Water molecules & 302 \\
\hline \multicolumn{2}{|l|}{ r.m.s deviaton from ideal } \\
\hline Bond lengths ( $\AA$ ) & 0.011 \\
\hline Bond angles (deg.) & 1.43 \\
\hline \multicolumn{2}{|l|}{ Ramachandran plot ${ }^{\mathrm{e}}$} \\
\hline Most favored regions (\%) & 91.7 \\
\hline Allowed regions (\%) & 8.3 \\
\hline \multicolumn{2}{|c|}{ 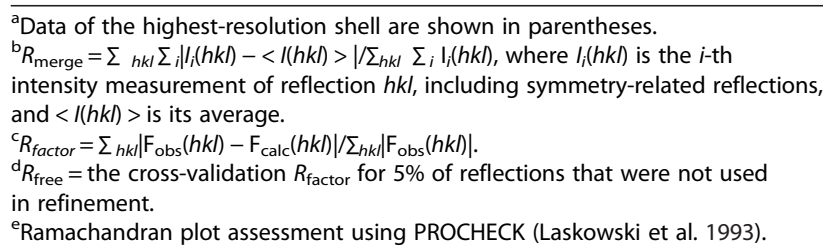 } \\
\hline
\end{tabular}

using XtalView (McRee 1999). The refined structure was assessed using PROCHECK (Laskowski et al. 1993). The figures were prepared with PyMOL (DeLano 2002). Energy minimization of the substrate-binding model was performed with GROMACS-4.6.3 (Hess et al. 2008) according to online tutorials (http://www.gromacs.org/).

\section{3-Quinuclidinone reductase assay of wild-type and mutant RrQR enzymes}

The reaction mixture comprised, in $2 \mathrm{ml}, 500 \mathrm{mM}$ 3-quinuclidinone $\cdot \mathrm{HCl}, 566 \mathrm{mM}$ glucose, $1.2 \mathrm{mg} / \mathrm{ml}$ $\mathrm{NAD}^{+}$or $\mathrm{NADP}^{+}, 0.4 \mathrm{mg} / \mathrm{ml}$ glucose dehydrogenase (Amano Enzymes, Nagoya, Japan), $200 \mathrm{mM}$ potassium phosphate buffer ( $\mathrm{pH}$ 7.0), and an appropriate amount of purified enzyme. After $20 \mathrm{~h}$ of incubation at $30^{\circ} \mathrm{C}$, the reaction was terminated by addition of an equal volume of saturated $\mathrm{Na}_{2} \mathrm{CO}_{3}$, and then the formed 
3-quinuclidinol was extracted with an equal volume of chloroform. The optical purity of 3-quinuclidinol was determined by gas-liquid chromatography analysis, as described previously (Uzura et al., 2009).

Mutations were introduced into the plasmid pET28a containing the $\mathrm{RrQR}$ gene. The mutant proteins were purified using a Ni-NTA column (Qiagen). The activities of the wild-type and mutant RrQR enzymes were analyzed as follows. The standard assay mixture was comprised of $2.5 \mathrm{ml}, 125 \mathrm{mM}$ 3-quinuclidinone- $\mathrm{HCl}, 350$ $\mu \mathrm{M}$ NADPH, and $200 \mathrm{mM}$ potassium phosphate buffer $(\mathrm{pH}$ 7.0), and the appropriate amount of the purified enzyme. After $2 \mathrm{~min}$ of incubation without the enzyme at $37^{\circ} \mathrm{C}$, the reaction was started by adding the enzyme, and then the decrease in absorbance at $340 \mathrm{~nm}$ due to NADPH oxidation was determined. One unit of RrQR activity was defined as the amount catalyzing the oxidation of $1 \mu \mathrm{mol}$ NADPH per min.

\section{Accession number}

Coordinates and structure factors have been deposited in the Protein Data Bank with accession number 4O0L.

\section{Results}

\section{Overall structure}

The crystal structure of RrQR was determined at 2.2- $\AA$ resolution and refined to a crystallographic $R$-factor of 0.192 and $R_{\text {free }}$ of 0.230 (Table 1 and Additional file 1 : Table S1). The asymmetric unit of the crystal contains four protomers of RrQR. The final model includes residues 7-272 for each protomer. There is no electron density for $\mathrm{N}$-terminus residues 1-6 in any of the four protomers, indicating that the region is flexible. The four protomers in the asymmetric unit are very similar to one another, with root-mean-square (r.m.s.) deviations of $0.20-0.24 \AA$. A clear electron density for NADPH is observed in each protomer, and the structures of the four NADPH molecules in the asymmetric unit exhibit similar conformations.

The crystal structure of $\operatorname{RQR}$ shows a tightly associated homotetramer (Figure 1a). The tetrameric structure is consistent with the results of a previous gel-filtration chromatographic analysis (Uzura et al. 2009). Like other SDR family proteins, the RrQR protomer can be divided into two domains: the core and small lobe structures (Figure $1 \mathrm{~b}$ and $\mathrm{c}$ ). The core structure exhibits the $\alpha / \beta$ Rossmann fold, with seven $\alpha$-helices, two $3_{10}$-helices and a central parallel $\beta$-sheet consisting of seven strands. The small lobe is away from the core, which lies between $\beta F$ and $\alpha G$ (residues 212-238) and contains one helix $\alpha F G$. A deep cleft exists between the core and the small lobe, and reaches to about the middle of the molecule. The NADPH cofactor is located at the bottom of the cleft. A remarkably extended structure is found in the N-terminal region, consisting of residues 7-19. There is a hydrophobic stacking of P10 with Y229 on $\alpha \mathrm{FG}$ of the neighboring protomer, which would contribute to the quaternary structural stability (Additional file 1: Figure S1).

The search for structurally homologous proteins was performed with the Dali server, and revealed several similar proteins: $\beta$-ketoacyl acyl carrier protein reductase (r.m.s. deviation of $1.2 \AA$ for $229 \mathrm{C} \alpha$ atoms (Fisher et al. 2000; PDB ID: 1edo)), trihydroxynaphthalene reductase (r.m.s. deviation of $1.2 \AA$ for $219 \mathrm{C} \alpha$ atoms (Liao et al. 2001; PDB ID: 1g0n)), and mannitol 2-dehydrogenase (r.m.s. deviation of $1.3 \AA$ for $234 \mathrm{C} \alpha$ atoms (Hörer et al. 2001; PDB ID: 1h5q)). Structural comparison showed that their core structures are almost superimposable, whereas remarkable structural diversities exist around the cleft (Figure 1c). The cleft is formed by the small lobe and a loop on the side opposite the lobe; this loop lies between $\beta E$ and $\alpha F$ (residues 171-178). In the small lobe (S-lobe), $\operatorname{RrQR}$ has one helix $\alpha \mathrm{FG}$, whereas the other enzymes each have two helices. Upon the loop, the mannitol 2-dehydrogenase has a small antiparallel $\beta$-sheet, and the other enzymes have different structures. Thus, we named the loop the variable loop (V-loop) (Figure 1c). The deep cleft is located above the B-face of the nicotinamide ring, and it has been described as the substrate-binding site in SDR family proteins. Therefore, the diverse structures around the cleft could enable the family proteins to accommodate various substrates.

\section{NADPH-binding mode of RrQR}

The bound NADPH is in an extended conformation, and the nicotinamide and adenine rings are about $10.5 \AA$ apart (Figure 2). Both ribose rings are in the $\mathrm{C}^{2}$ '-endo conformation. The adenine ring is in the anti conformation, and the nicotinamide ring is in the syn conformation. The conformation and the space above the nicotinamide ring allow the 4-pro-S hydride transfer from the B-side of the nicotinamide ring. The enzyme interacts with the NADPH through hydrogen bonds (Additional file 1: Figure S2). Side chains of the residues R60, S61, E86, Y181, K185, and T215 form hydrogen bonds with the NADPH molecule, and a side chain of N113 forms a hydrogen bond with a water molecule that is hydrogen-bonded to the NADPH (Figure 2 and Additional file 1: Figure S2). Among these residues, R60 and $\mathrm{S} 61$ form hydrogen bonds with the 2 '-phosphate group of the NADPH; these bonds are involved in the discrimination of NADPH from NADH. The main chains of the other residues, G35, G37, G39, G41, L58, A62, V87, T165, P210, and G211, also form hydrogen bonds with the NADPH molecule.

\section{Structure of the active site}

The active site of SDR enzymes is composed of a triad of catalytically important residues: serine, tyrosine, and 


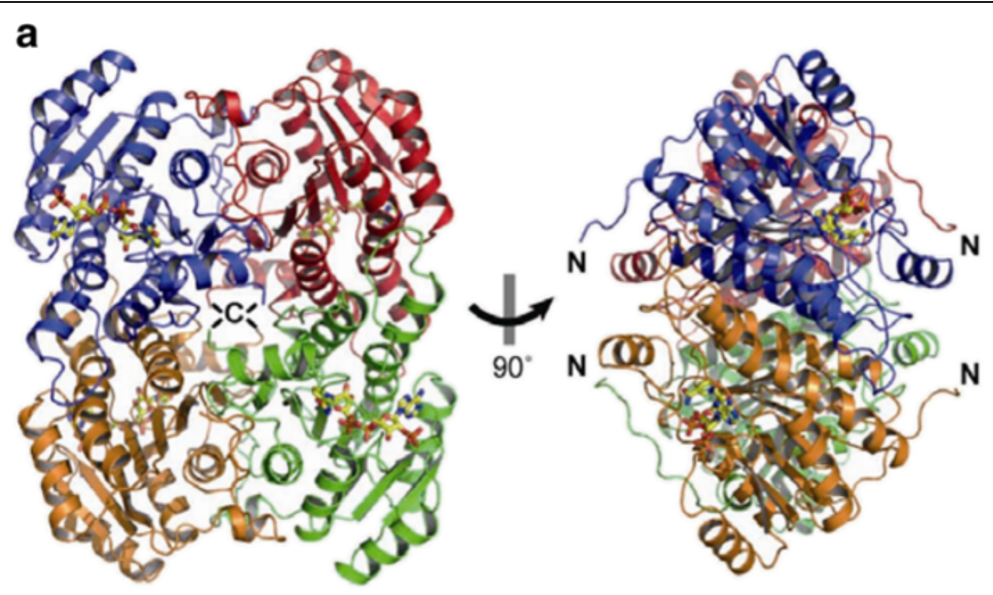

b
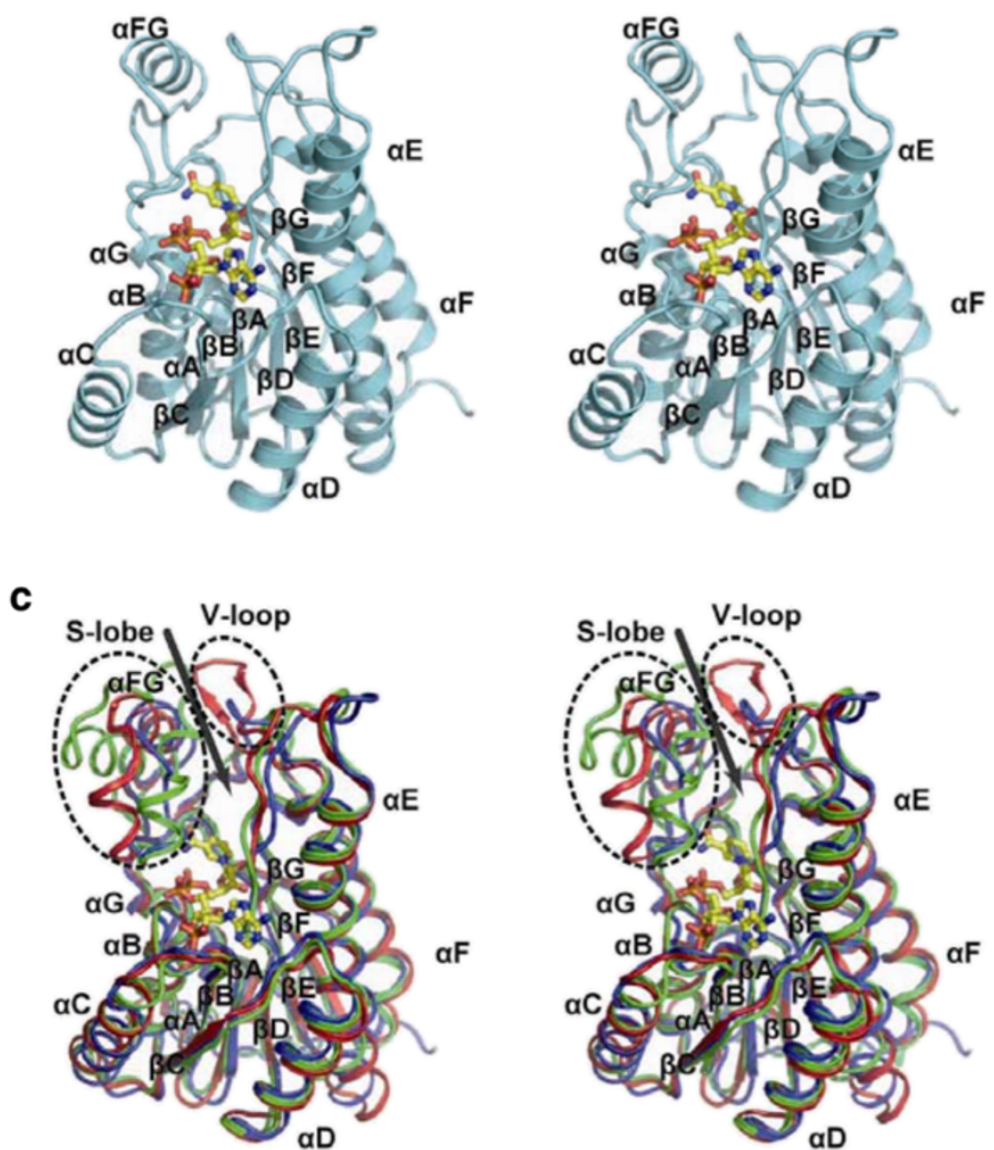

Figure 1 Overall structure of RrQR. a Overall view of RrQR. The protomers are shown in blue, red, orange, and green; NADPH molecules are yellow (left). Side view of RrQR (right). b Stereoview of the structure of the RrQR protomer, presented in light blue. The NADPH molecule is shown as a yellow stick model. c Stereoview of the protomer structure of RrQR, and superimposition with similar structures. RrQR, mannitol 2-dehydrogenase (PDB ID: 1h5q), and trihydroxynaphthalene reductase (PDB ID: 1g0n) are shown in blue, red, and green, respectively. The NADPH molecule of RrQR is shown as a yellow stick model. The a-helices, $a A-a G$, and the $\beta$-strands, $\beta A-\beta G$, are labeled. The aFG between aF and aG is also labeled. The small lobe and variable loop are encircled by dotted lines. The deep cleft is shown by a gray arrow. 

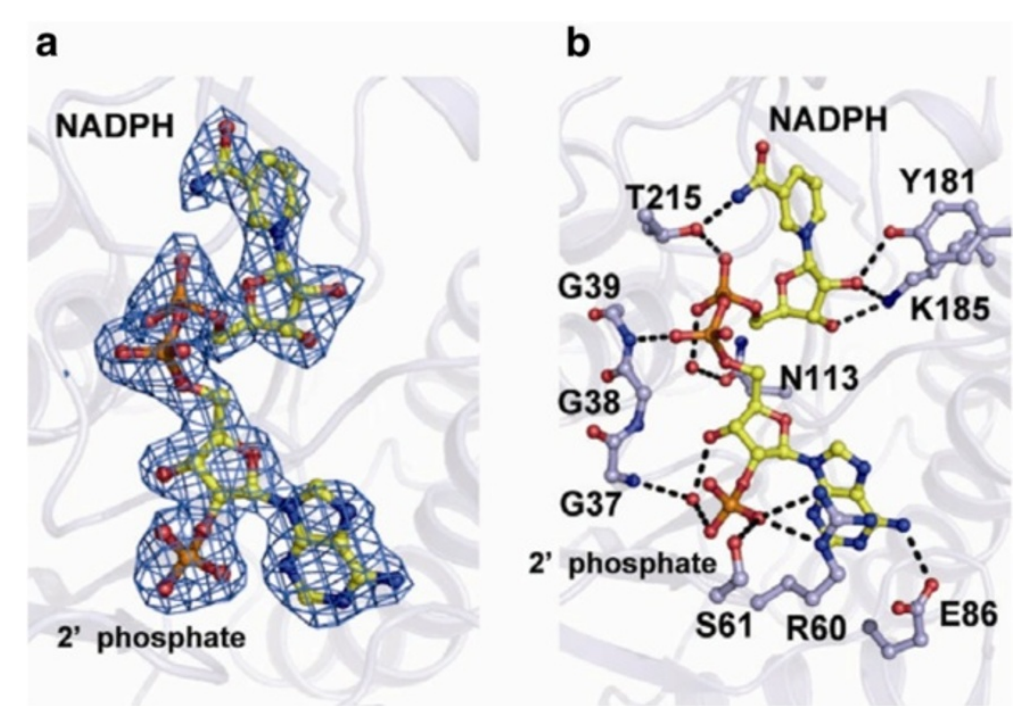

Figure 2 The NADPH-binding site of RrQR. a The NADPH molecule is represented by a stick model, and the NADPH omit $F_{\mathrm{O}}-F_{\mathrm{C}}$ map contoured at $3.0 \sigma$ is shown in light blue. There is no cutoff of the radius of the atoms for the display of the electron density. $\mathbf{b}$ Side chains of the amino acid residues forming hydrogen bond(s) with the NADPH molecule are represented by a stick model, and the hydrogen bonds are shown by dotted lines. Residues G37 through G39 are also shown by a stick model.

lysine (Jörnvall et al. 1995). More recently, an asparagine residue or a histidine residue at the corresponding position was added to the catalytic triad (Filling et al. 2002; Kubota et al. 2011), since a water molecule bound to the main-chain carbonyl group of the asparagine or histidine is assumed to participate in the reductive reaction. The configuration of these catalytic tetrad residues is well superimposed on that of RrQR (Figure 3a), and thus the catalytic reaction would be achieved through the same proton relay (Filling et al. 2002).

To identify the catalytic site where the carbonyl group of 3-quinuclidinone is converted to the hydroxyl group, we performed a mutation analysis of $\mathrm{RrQR}$. In this reductase, residues N138, S166, Y181, and K185 correspond to the catalytic tetrad. Mutations of these residues, S166A, Y181A, Y181F, and K185A, resulted in a complete loss of activity (Table 2). The results reveal the catalytic residues of $\mathrm{RrQR}$, providing a basis for the reaction-based model as described below.

\section{Substrate-binding site and 3-quinuclidinone-binding model}

A small space is found above the B-face of the nicotinamide ring, and is assumed to be the substrate-binding site. This small pocket, with a diameter of $7 \AA$ and a depth of $8 \AA$, is formed by S166, I167, S168, N173, Q178, Y181, and F212 (Figure 3b). None of these residues is charged, and the most notable characteristic of the pocket is a hydrophobic wall composed of I167 and F212, located at about the bottom of the pocket.
To obtain further insights into the mechanism underlying the RrQR reaction, we built a substrate-binding model that ensures a stereospecific reaction (Figure 4). Structural analyses of TR-II, a member of the SDR family, revealed that the catalytic reaction is achieved through a slight rotational movement of the substrate molecule (Yamashita et al. 1999; Yamashita et al. 2003). The chemical reaction catalyzed by the enzymes TR-II and $\mathrm{RrQR}$ is the same, and involves the reduction of a carbonyl group to a hydroxyl group. Superimposition of their structures shows that the configurations of the catalytic residues (S166, Y181, and K185) and the nicotinamide ring of NADPH are essentially identical (Figure 3a). This structural similarity leads to the rational substrate-binding model of $\mathrm{RrQR}$ based on the complex structure of the TR-II-substrate complex.

The ketone group of the substrates has a trigonal planar configuration, with $\mathrm{C}-\mathrm{C}-\mathrm{O}$ and $\mathrm{C}-\mathrm{C}-\mathrm{C}$ bond angles of about $120^{\circ}$. Therefore, the superimposition of the ketone group (C-CO-C) of 3-quinuclidinone to that of tropinone can be limited to two patterns, which are related by a $180^{\circ}$ rotation about the carbonyl $\mathrm{C}-\mathrm{O}$ bond axis (Figure $4 \mathrm{a}$ and $\mathrm{b}$ ). The reaction is achieved through the proton transfer to the carbonyl group of a substrate from the catalytic residue tyrosine, and through the nucleophilic attack of the hydride of NADPH on the carbonyl carbon of the substrate. Thereby, the hydroxyl group of the product relocates to the side opposite that of the nicotinamide ring. The geometry presented in Figure 4a agrees with the catalytic reaction of $\mathrm{RrQR}$, producing ( $R$ )-3-quinuclidinol from 3-quinuclidinone. 


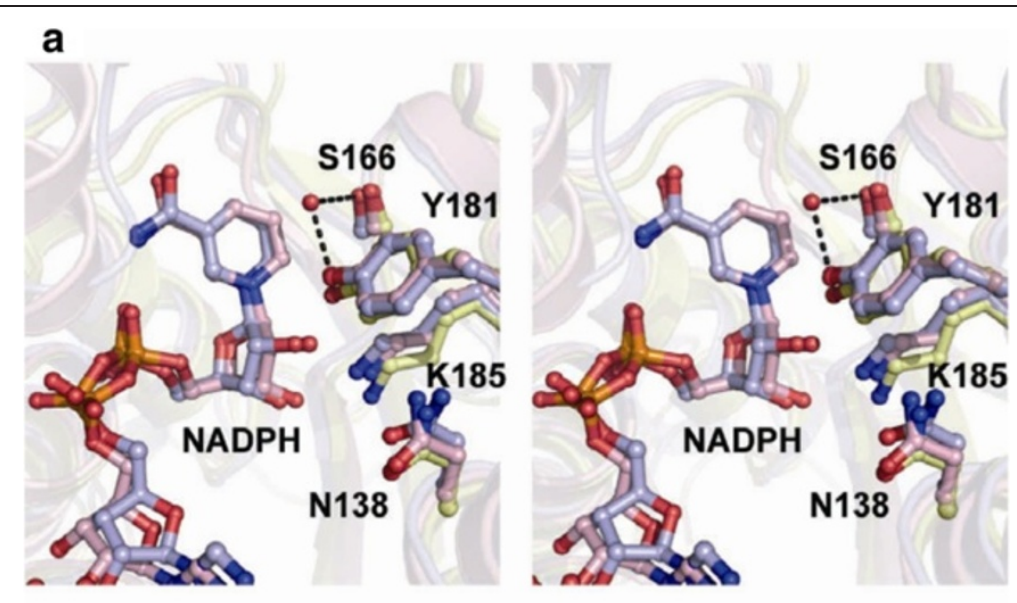

b
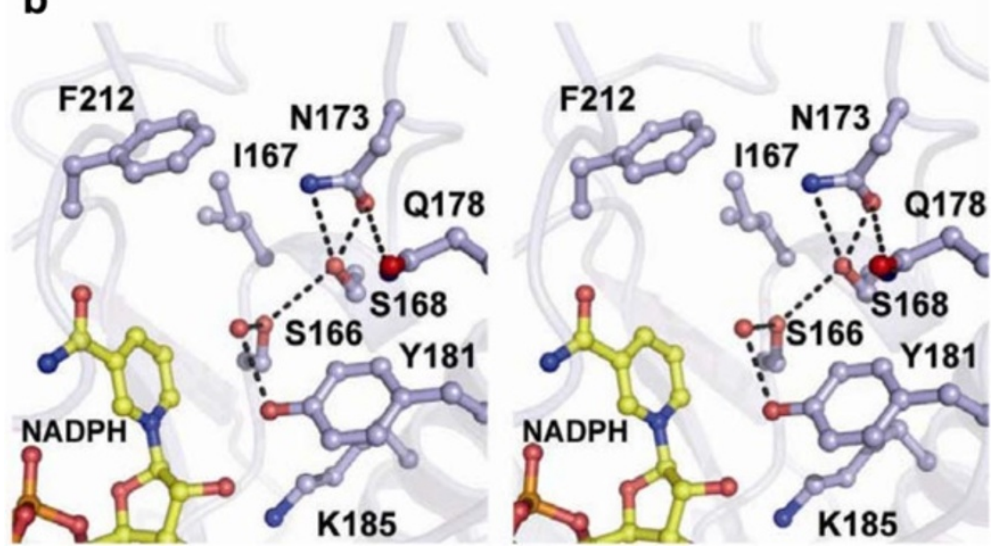

Figure 3 The active site of RrQR. a Stereo view of the active site of RrQR and its superimposition with those of TR-II and $3 \beta / 17 \beta$-hydroxysteroid

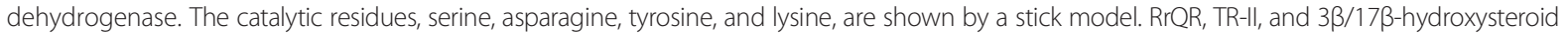
dehydrogenase are shown in light blue, pink, and pale yellow, respectively. The residues of RrQR are labeled. $\mathbf{b}$ Stereo view of the substrate-binding site of RrQR. A water molecule in the active site is shown as a sphere, and hydrogen bonds are shown by dotted lines. The hydrophobic wall is comprised of 1167 and F212.

Table 2 Quinuclidinone reductase activities of the wild-type and mutant RrQR enzymes

\begin{tabular}{|c|c|c|c|c|c|}
\hline & Mutation & $\begin{array}{l}\text { Relative activity for } \\
\text { 3-qinuclidinone }\end{array}$ & $K_{\mathrm{m}}(\mathrm{mM})^{\mathrm{b}}$ & $V_{\max }(\text { unit } / \mathrm{mg})^{\mathrm{a}}$ & $k_{\text {cat }}\left(\sec ^{-1}\right)$ \\
\hline & WT & 100 & 440 & 31.2 & 15.1 \\
\hline \multirow[t]{4}{*}{ Catalytic site } & $\mathrm{S} 166 \mathrm{~A}$ & ND & & & \\
\hline & Y181A & ND & & & \\
\hline & Y181F & ND & & & \\
\hline & K185A & ND & & & \\
\hline \multirow[t]{7}{*}{ Substrate-binding site } & I167A & ND & & & \\
\hline & $1167 \mathrm{~V}$ & 24.5 & 308 & 5.56 & 2.69 \\
\hline & S168A & 115.7 & 168 & 19.9 & 9.64 \\
\hline & $\mathrm{N} 173 \mathrm{~A}$ & 97.8 & 100 & 11.6 & 5.62 \\
\hline & Q178A & 164.5 & 103 & 20.2 & 9.79 \\
\hline & F212A & ND & & & \\
\hline & $\mathrm{F} 212 \mathrm{~L}$ & ND & & & \\
\hline
\end{tabular}

ND: not detected.

${ }^{a}$ The analyses were performed in the presence of $125 \mathrm{mM}$ 3-quinuclidinone.

${ }^{\mathrm{b}}$ The analyses were performed in the presence of $350 \mu \mathrm{M}$ NADPH. 

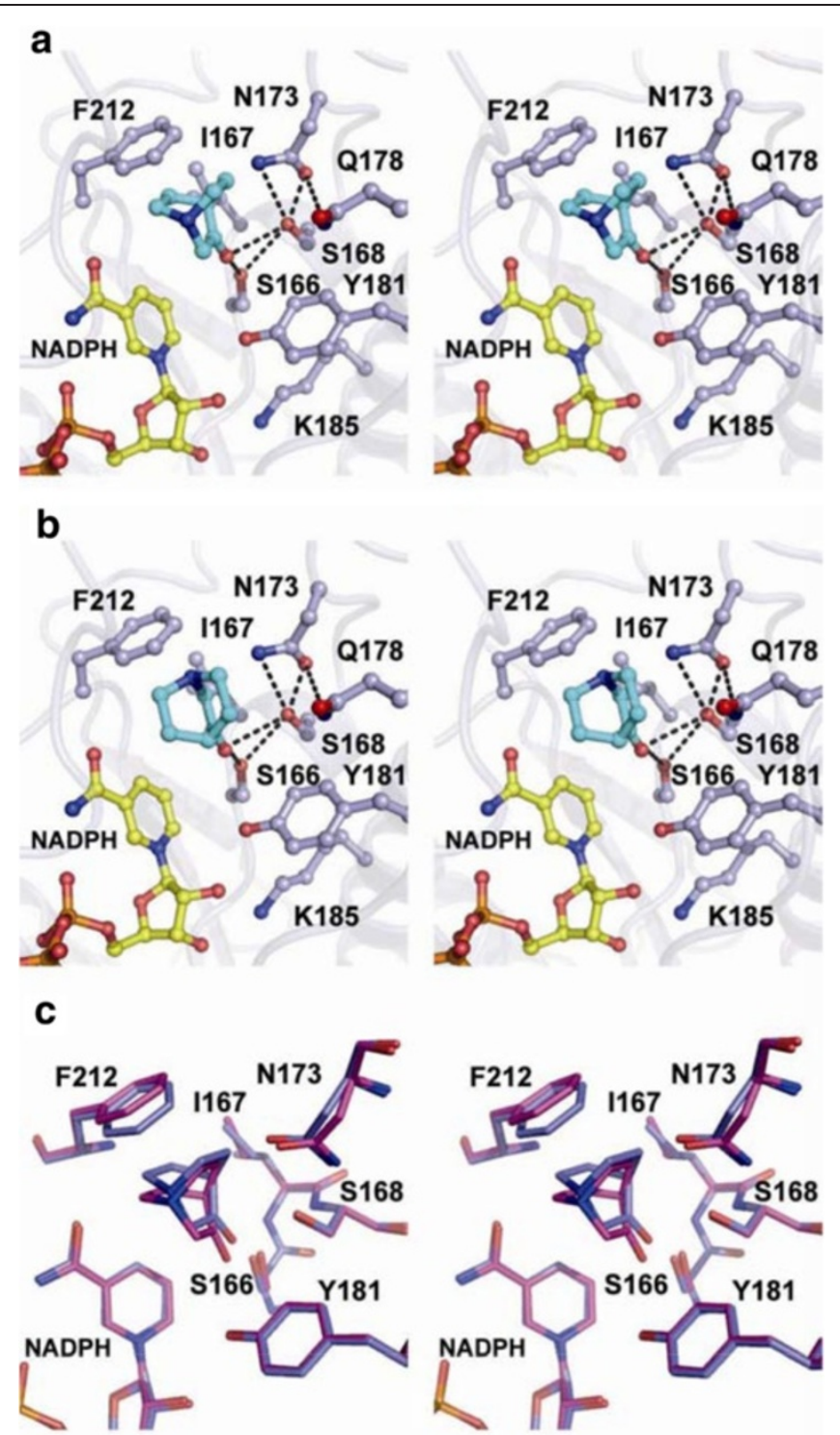

d

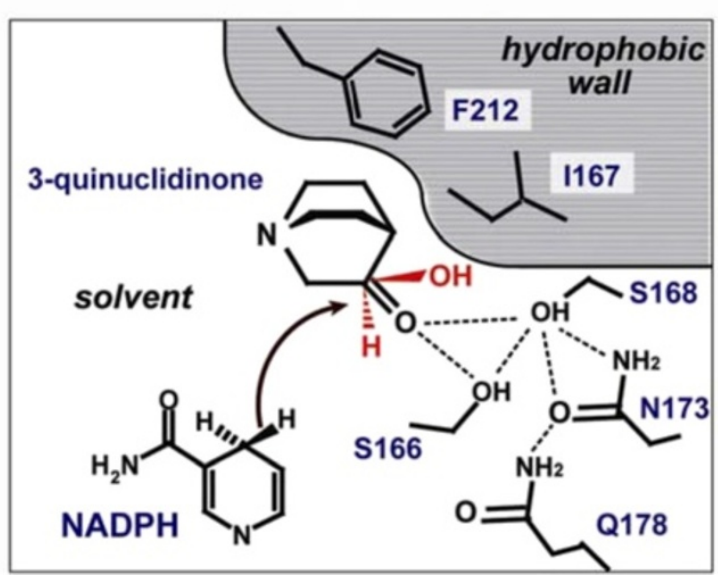

Figure 4 (See legend on next page.) 
(See figure on previous page.)

Figure 4 Stereospecific binding for the asymmetric reduction of 3-quinuclidinone by RrQR. A 3-quinuclidinone in the active site of RrQR is shown by a stick model and in cyan. a The catalytic model of 3-quinuclidinone-RrQR ensures the product (R)-3-quinuclidinol. The hydrophobic wall comprised of 1167 and F212 is adjacent to the hydrophobic side of the substrate, which stabilizes the substrate binding. The tertiary amine group of the substrate is exposed to solvent. $\mathbf{b}$ The noncatalytic model of 3-quinuclidinone-RrQR leads to the product (S)-3-quinuclidinol. The hydrophobic wall is adjacent to the amine group of the substrate, resulting in an unstable state. A hydrophobic surface of the substrate is exposed to solvent. c The refined structure of RrQR by an energy minimization (red) is superimposed on the structure of the catalytic model shown in Figure $4 \mathbf{a}$ (blue). $\mathbf{d}$ Schematic diagram of the catalytic mechanism of RrQR. The hydride transfer from NADPH to the carbonyl carbon of 3-quinuclidinone is depicted by an arrow. The hydrophobic wall comprised of I167 and F212 is adjacent to the hydrophobic surface of the substrate. The hydrogen bonds in the active site are shown by dotted lines. The hydroxyl group of the product is shown in red.

On the other hand, the geometry in Figure $4 \mathrm{~b}$ results in the production of another stereoisomer, $(S)$-3-quinuclidinol, which does not agree with the catalytic reaction of RrQR. The enzyme RrQR produces only $(R)$-3-quinuclidinol. Thus we describe the geometry in Figure $4 \mathrm{a}$ as a catalytic model and that in Figure $4 \mathrm{~b}$ as a noncatalytic model.

We then evaluated the surroundings of the active site for the two models. The molecule 3-quinuclidinone contains a tertiary amine group, which has basic and hydrophilic properties due to a lone electron pair. In the catalytic model, the amine group is exposed to solvent, while on the opposite side, hydrophobic hydrocarbons of 3-quinuclidionone are buried in the small pocket and adjacent to the hydrophobic wall composed of I167 and F212, resulting in a stable state. On the other hand, in the noncatalytic model, the amine group is located adjacent to a hydrophobic wall composed of I167 and F212, and the hydrocarbons of 3-quinuclidinone are exposed to the solvent, thereby resulting in an unstable state. The differences between the two models can explain how the RrQR enzyme produces $(R)$-3-quinuclidinol prior to $(S)$-3-quinuclidinol from 3-quinuclidinone.

\section{Critical residues in the substrate-binding site}

The purified RrQR enzyme catalyzed the production of $(R)$-quinuclidinol from 3-quinuclidinone, with an enantiomeric excess of $>99.9 \%$ (Uzura et al. 2009). The residues interacting with the substrate were predicted by the program Ligplot (Laskowski and Swindells 2011, Additional file 1: Figure S3), and Table 2 summarizes the results of the mutation analysis of the substrate-binding site. The analysis revealed that alanine mutations at hydrophobic residues I167 and F212 significantly affect the activity. The F212A and F212L mutants completely lost their catalytic activity, indicating the importance of the bulky hydrophobic property of phenylalanine. The accessible surface areas of phenylalanine and leucine are $30.9 \AA^{2}$ and $26.9 \AA^{2}$, respectively. Therefore, the bulky hydrophobic surface generated from the additional hydrocarbons plays a significant role in the activity. Similarly, the I167A mutant lost the activity, and the I167V mutant showed a significant decrease in the activity, to $24.5 \%$ of the wild type. With respect to the catalytic model presented in Figure 4a, the hydrocarbons of I167 and F212 would have direct hydrophobic interactions with the substrate, because the distances between the substrate and the side chains of I167 or F212 are within $4 \AA$.

In contrast to the mutations at the hydrophobic residues I167 and F212, the mutations at polar amino acid residues S168, N173 and Q178 did not exhibit significantly reduced activity (Table 2 ). In these mutations, the $K_{\mathrm{m}}$ values are decreased and the $k_{\text {cat }}$ values are decreased, meaning that the affinities of the mutants are increased but the rates of the catalysis are decreased. The higher affinities of S168A, N173A and Q178A may be due to the increase of hydrophobicity favorable for the substrate binding. The reductions of the $k_{\text {cat }}$ value of these mutants suggest that the hydrogen network including catalytic residue S166 may be favorable for the reaction (Figure 4).

We subsequently refined the structure of the substratebinding model by means of energy minimization, indicating that slight movements of residues I167, N173 and F212 enable the enzyme to adapt to 3-quinuclidinone (Figure 4c). Therefore, the active site of the enzyme has a preference for the substrate without large structural rearrangements.

The residues forming the substrate-binding pocket are located in the small lobe (residue F212) and on or near the variable loop (residues I167, S168, N173, and Q178). The two structural elements, the small lobe and the V-loop, are characteristic structural regions of the RrQR enzyme. Therefore, the stereospecific catalytic activity of RrQR would be achieved by this novel substrate-binding mechanism (Figure 4d).

\section{Discussion}

The structure of RrQR is the first structure of quinuclidinone reductase shown to catalyze the stereo-specific reduction of 3-quinuclidinone. The RrQR enzyme produces $(R)$-3-quinuclidinol with more than $99.9 \%$ enantiomeric excess (Uzura et al. 2009). The angles of the hydride transfer reaction $(\mathrm{C}-\mathrm{H}-\mathrm{C})$ have been suggested to be rigidly restricted (Wu and Houk 1987a; Wu and Houk 1987b). However, the structural model indicates that RrQR recognizes the substrate within a narrow area (Figure 4). In agreement with this, the $K_{m}$ value for 
3-quinuclidinone $(440 \mathrm{mM})$ is relatively high. Therefore, the RrQR enzyme would bind loosely to the substrate, but the substrate-binding site is sufficient to accommodate 3 -quinuclidinone in one direction to produce the $R$-form of 3-quinuclidinol.

The substrate orientation is restricted by the circumstances of the catalytic site of RrQR. The substratebinding pocket of RrQR is largely formed by the hydrophobic wall. The surface of the 3-quinuclidinone is divided into two parts, a hydrophilic face and hydrophobic face. Thus, the asymmetric reduction of 3-quinuclidinone is achieved by the ability of RrQR to discriminate the hydrophobic surface from the hydrophilic surface of 3-quinuclidinone. The hydrophobic residues of I167 and F212 function not only in the stereospecific substratebinding but also in the substrate accommodation leading to the catalytic reaction (Table 2), and therefore we could not analyze the effect of the mutagenesis on the stereoselectivity. Several compounds, including 4-chloro-3-oxobutanoate and ketopantoyl lactone, are catalyzed by $\operatorname{RQR}$, but the optical purities of the products are reduced (Uzura et al. 2009). Therefore, the size and the chemical property of the catalytic pocket of $\operatorname{RQR}$ is suitable for the binding of 3-quinuclidinone in the specific orientation.

Structural and mutation analyses of TR enzymes have indicated that the enzymes utilize electrostatic interactions between an amine group of the substrate and the carboxyl group of a Glu residue to determine the substrate-binding orientations (Nakajima et al. 1998; Nakajima et al. 1999). Similarly, levodione reductase also utilizes the electrostatic interaction between a positively charged surface of the substrate and a Glu residue to bind the substrate in the specific orientation (Sogabe et al. 2003). In contrast, the RrQR enzyme retains the stereospecific activity exhibited by the hydrophobic wall. Therefore, RrQR possesses a novel mechanism for the stereospecific reaction led by a characteristic interaction, in which the hydrophobicity of I167 and F212 plays a crucial role in the stereospecific reduction of 3-quinuclidinone.

In conclusion, we have described the structural analysis of an enzyme that catalyzes the stereospecific reduction of 3-quinuclidinone to (R)-3-quinuclidinol. The three-dimensional substrate-binding model and the mutation analysis indicate that the specific substratebinding would be achieved through the interactions between hydrophobic residues and a hydrophobic substrate surface. The hydrophobic interactions would be crucial for the asymmetric reduction of 3-quinuclidinone. The reason for the low affinity for the substrate is that the compound 3-quinuclidinone is not the natural substrate for the enzyme. Therefore, it may be possible that tuning of the enzyme by genetic strategies and/or optimization of the reaction condition will yield an effective system for the expression of its maximum activity. The structural insights uncovered by this study will provide valuable information for investigations of enzymes that catalyze small cyclic molecules in specific orientations.

\section{Additional file}

Additional file 1: Supplementary data associated with this article can be found in the online version.

\section{Competing interests}

The authors declare that they have no competing interests.

\section{Acknowledgments}

We thank the Beamline staff at Photon Factory for data collection. This work was performed with the approval of the Photon Factory Advisory Committee, Japan (Proposal No. 200852-001) and supported by the Targeted Proteins Research Program (TPRP) from the Ministry of Education, Culture, Sports, Science, and Technology of Japan.

\section{Author details}

${ }^{1}$ Department of Applied Biological Chemistry, Graduate School of Agricultural and Life Sciences, University of Tokyo, 1-1-1 Yayoi, Bunkyo-ku, Tokyo 113-8657, Japan. ${ }^{2}$ Division of Applied Life Sciences, Graduate School of Agriculture, Kyoto University, Kitashirakawa-Oiwakecho, Sakyo-ku, Kyoto 606-8502, Japan. ${ }^{3}$ Division of Applied Life Sciences, Graduate School of Life and Environmental Sciences, Osaka Prefecture University, 1-1 Gakuencho, Naka-ku, Sakai, Osaka 599-8531, Japan. ${ }^{4}$ Research and Development Center, Nagase \& Co., Ltd., 2-2-3 Murotani, Nishi-ku, Kobe 651-2241, Japan. ${ }^{5}$ Faculty of Bioenvironmental Science, Kyoto Gakuen University, Sogabe-cho, Kameoka 621-8555, Japan.

Received: 14 December 2013 Accepted: 28 December 2013 Published: 7 February 2014

\section{References}

Alabaster VA (1997) Discovery \& development of selective M3 antagonists for clinical use. Life Sci 60:1053-1060

Bietti G, Doods HN, Cereda E, Schiavone A, Donetti A, Giovanni B (1990) New $R(-) 3$-quinuclidinol derivatives. EP 404737

Bossard P (1997) Verfahren zur Gewinnung von (R)-3-Chinuclidinol durch Racematspaltung von (R,S)-3-Chinuclidinol. DE 19715465

Brieden W (1998) Process for the preparation of optically active 3-quinuclidinol. US Patent 5:744,606

Brünger AT, Adams PD, Clore GM, DeLano WL, Gros P, Grosse-Kunstleve RW, Jiang JS, Kuszewski J, Nilges M, Pannu NS, Read RJ, Rice LM, Simonson T, Warren GL (1998) Crystallography \& NMR system: a new software suite for macromolecular structure determination. Acta Crystallogr D Biol Crystallogr 54:905-921

Cross PE, Stobie A (1993) Quinuclidine esters process and intermediate for their preparation and pharmaceutical compositions containing them. WO 9306098

DeLano WL (2002) The PyMOL User's Manual. DeLano Scientific, San Carlos, CA

Filling C, Berndt KD, Benach J, Knapp S, Prozorovski T, Nordling E, Ladenstein R, Jörnvall H, Oppermann U (2002) Critical residues for structure and catalysis in short-chain dehydrogenases/reductases. J Biol Chem 277:25677-25684

Fisher M, Kroon JT, Martindale W, Stuitje AR, Slabas AR, Rafferty JB (2000) The $X$-ray structure of Brassica napus $\beta$-keto acyl carrier protein reductase and its implications for substrate-binding and catalysis. Structure 8:339-347

Goldberg K, Schroer K, Lütz S, Liese A (2007) Biocatalytic ketone reduction-a powerful tool for the production of chiral alcohols-part l: processes with isolated enzymes. Appl Microbiol Biotechnol 76:237-248

Hess B, Kutzner C, van der Spoel D, Lindahl EJ (2008) GROMACS 4: algorithms for highly efficient, load-balanced, and scalable molecular simulation. J Chem Theory Comput 4:435-447

Hörer S, Stoop J, Mooibroek H, Baumann U, Sassoon J (2001) The crystallographic structure of the mannitol 2-dehydrogenase $\mathrm{NADP}^{+}$binary complex from Agaricus bisporus. J Biol Chem 276:27555-27561

Hummel W (1997) New alcohol dehydrogenases for the synthesis of chiral compounds. Adv Biochem Eng Biotechnol 58:145-184 
Ishihara T, Kakuta H, Moritani H, Ugawa T, Yanagisawa I (2004) Synthesis and biological evaluation of quinuclidine derivatives incorporating phenothiazine moieties as squalene synthase inhibitors. Chem Pharm Bull (Tokyo) 52:1204-1209

Jörnvall H, Persson B, Krook M, Atrian S, Gonzàlez-Duarte R, Jeffery J, Ghosh D (1995) Short-chain dehydrogenases/reductases (SDR). Biochemistry 34:6003-6013

Kataoka M, Kita K, Wada M, Yasohara Y, Hasegawa J, Shimizu S (2003) Novel bioreduction system for the production of chiral alcohols. Appl Microbiol Biotechnol 62:437-445

Kubota K, Nagata K, Okai M, Miyazono K, Soemphol W, Ohtsuka J, Yamamura A, Saichana N, Toyama H, Matsushita K, Tanokura M (2011) The crystal structure

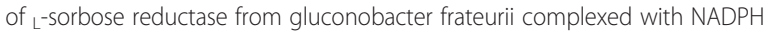
and L-sorbose. J Mol Biol 407:543-555

Laskowski RA, Swindells MB (2011) LigPlot+: multiple ligand-protein interaction diagrams for drug discovery. J Chem Inf Model 51:2778-2786

Laskowski RA, MacArthur MW, Moss DS, Thornton JM (1993) PROCHECK - a program to check the stereochemical quality of protein structures. J Appl Crystallogr 26:283-291

Leusch A, Tröger W, Greischel A, Roth W (2000) Pharmacokinetics of the M1-agonist talsaclidine in mouse, rat, rabbit and monkey, and extrapolation to man. Xenobiotica 30:797-813

Liao D, Basarab GS, Gatenby AA, Valent B, Jordan DB (2001) Structures of trihydroxynaphthalene reductase-fungicide complexes: implications for structure-based design and catalysis. Structure 9:19-27

Matsuyama A, Hamatani T (1998) Processes for production of optical active quinuclidinol. EP 863212

McCoy AJ, Grosse-Kunstleve RW, Adams PD, Winn MD, Storoni LC, Read RJ (2007) Phaser crystallographic software. J Appl Cryst 40:658-674

McRee DE (1999) XtalView/Xfit-a versatile program for manipulating atomic coordinates and electron density. J Struct Biol 125:156-165

Muchmore DC (1993) Enantiomeric enrichment of (R, S)-quinuclidinol. US Patent $5: 215,918$

Murshudov GN, Vagin AA, Dodson EJ (1997) Refinement of macromolecular structures by the maximum-likelihood method. Acta Crystallogr D Biol Crystallogr 53:240-255

Nakajima K, Yamashita A, Akama H, Nakatsu T, Kato H, Hashimoto T, Oda J, Yamada Y (1998) Crystal structures of two tropinone reductases: different reaction stereospecificities in the same protein fold. Proc Natl Acad Sci USA 95:4876-4881

Nakajima K, Kato H, Oda J, Yamada Y, Hashimoto T (1999) Site-directed mutagenesis of putative substrate-binding residues reveals a mechanism controlling the different stereospecificities of two tropinone reductases. J Biol Chem 274:16563-16568

Nomoto F, Hirayama Y, Ikunaka M, Inoue T, Otsuka K (2003) A practical chemoenzymatic process to access (R)-quinuclidin-3-ol on scale. Tetrahedron Asymmetry 14:1871-1877

Oppermann U, Filling C, Hult M, Shafqat N, Wu X, Lindh M, Shafqat J, Nordling E, Kallberg Y, Persson B, Jörnvall H (2003) Short-chain dehydrogenases/reductases (SDR): the 2002 update. Chem Biol Interact 143-144:247-253

Otwinowski Z, Minor W (1997) Processing of X-ray diffraction data collected in oscillation mode. Methods Enzymol 276:307-326

Rehavi M, Maayani S, Sokolovsky M (1977) Enzymatic resolution and cholinergic properties of (+/-)3-quinuclidinol derivatives. Life Sci 21:1293-1302

Rzeszotarski WJ, McPherson DW, Ferkany JW, Kinnier WJ, Noronha-Blob L, Kirkien-Rzeszotarski A (1988) Affinity and selectivity of the optical isomers of 3-quinuclidinyl benzilate and related muscarinic antagonists. J Med Chem 31:1463-1466

Sato E, Enomoto K (1999) Process for producing optically active 3-quinuclidinol derivatives. EP 945518

Sogabe S, Yoshizumi A, Fukami TA, Shiratori Y, Shimizu S, Takagi H, Nakamori S, Wada M (2003) The crystal structure and stereospecificity of levodione reductase from Corynebacterium aquaticum M-13. J Biol Chem 278:19387-19395

Stembach LH, Kaiser S (1952) Antispasmodics. I. Bicyclic basic Alcohols. J Am Chem Soc 74:2215-2218

Takenaka M (2006) Process for producing optically active 3-quinuclidinols. US Patent 20060047122

Takeshita D, Kataoka M, Miyakawa T, Miyazono Kl, Uzura A, Nagata K, Shimizu S, Tanokura M (2009) Crystallization and preliminary X-ray analysis of the $\mathrm{NADPH}$-dependent 3-quinuclidinone reductase from Rhodotorula rubra. Acta Crystallogr Sect F 65:645-647
Takeuchi M, Naito R, Hayakawa M, Okamoto Y, Yonetoku Y, Ikeda K, Isomura Y (1996) EP 0801067

Uzura A, Nomoto F, Sakoda A, Nishimoto Y, Kataoka M, Shimizu S (2009) Stereoselective synthesis of ( $R$ )-3-quinuclidinol through asymmetric reduction of 3-quinuclidinone with 3-quinuclidinone reductase of Rhodotorula rubra. Appl Microbiol Biotechnol 83:617-626

Ward JS, Merritt L, Calligaro DO, Bymaster FP, Shannon HE, Mitch CH, Whitesitt C, Brunsting D, Sheardown MJ, Olesen PH, Swedberg MD, Jeppesen L, Sauerberg P (1998) 1,2,5-Thiadiazole analogues of aceclidine as potent $\mathrm{m} 1$ muscarinic agonists. J Med Chem 41:379-392

Wu YD, Houk KN (1987a) Transition structures for hydride transfers. J Am Chem Soc 109:906-908

Wu YD, Houk KN (1987b) Theoretical transition structures for hydride transfer to methyleneiminium ion from methylamine and dihydropyridine. On the nonlinearity of hydride transfers J Am Chem Soc 109:2226-2227

Yamamoto H, Ueda M, Ritsuzui P, Hamatani T (2003) Methods for producing optical active alcohols. EP 1318200

Yamashita A, Kato H, Wakatsuki S, Tomizaki T, Nakatsu T, Nakajima K, Hashimoto T, Yamada Y, Oda J (1999) Structure of tropinone reductase-II complexed with $\mathrm{NADP}^{+}$and pseudotropine at $1.9 \AA$ resolution: implication for stereospecific substrate binding and catalysis. Biochemistry 38:7630-7637

Yamashita A, Endo M, Higashi T, Nakatsu T, Yamada Y, Oda J, Kato H (2003) Capturing enzyme structure prior to reaction initiation: tropinone reductase-II-substrate complexes. Biochemistry 42:5566-5573

doi:10.1186/2191-0855-4-6

Cite this article as: Takeshita et al: Structural basis of stereospecific reduction by quinuclidinone reductase. AMB Express 2014 4:6.

\section{Submit your manuscript to a SpringerOpen ${ }^{\odot}$ journal and benefit from:}

- Convenient online submission

Rigorous peer review

- Immediate publication on acceptance

- Open access: articles freely available online

- High visibility within the field

- Retaining the copyright to your article

Submit your next manuscript at springeropen.com 